\title{
A Quantum Approach to the Psychosomatic Phenomenon: Co-Emergence and Time Entanglement of Mind and Matter
}

\author{
Pierre UZAN
}

uzanpier@gmail.com

\begin{abstract}
This paper explores the philosophy of neutral monism within the framework of a generalized version of quantum theory where all references to the physical world have been relaxed. Psychic and somatic features of the individual are conceived of as co-emergent, complementary properties of an underlying, psychophysical level of reality. It is shown that their entanglement can be interpreted in terms of non-causal correlations and parametrized by time, which thus plays the role of psychophysical interface.
\end{abstract}

Key words: psychosomatic unity, co-emergence, complementarity, time-entanglement

\section{Introduction}

As shown by Bohm and Hiley (1993) and, more recently, by Atmanspacher, Römer and Walach (2002) and by Primas (2003, 2010), the key quantum concepts of complementarity and entanglement can be exported beyond the strict framework of quantum physics and even beyond the material realm. In particular, as suggested by these authors, the concepts of complementarity and entanglement, which are indeed intimately linked, can be fruitfully used to tackle the mind-body problem. Following this line of thought, this paper suggests a modelling of the psychosomatic phenomenon within the framework of a generalized quantum theory where all particular references to the physical world have been relaxed. In such a theory, the notion of "system" is defined as any part of reality which is an object of attention and investigation and that of "observable" as any meaningful property which can be measured or observed in a reproducible way.

Section I is an attempt to highlight the essential characteristics of the phychosomatic phenomenon. This phenomenon essentially relies on a thorough entanglement between the psychic and the organic features of the individual -hence the notion of "psychosomatic unity". It is suggested that their correlations can be conceived of as remnants of their common origin and do not consequently require the existence of causal connections to be explained. 
The co-emergence of the complementary, mental and physiological aspects of the individual from the psychophysical unity where they are enfolded is understood as an epistemic process. Section II presents the main propositions to deal with the mind-body problem and focuses on the philosophy of neutral monism originally proposed by Spinoza. As will be explained, neutral monism seems to provide the ideal framework to tackle this problem along the line of thought developed in section I. Section III explores the possibility of exporting the concepts of complementarity and entanglement beyond the strict framework of quantum physics where they are rigorously defined, and even beyond the material realm to represent the psychosomatic phenomenon. A generalisation of Bell's inequalities proposed by Landeau (1987) will be used in order to establish a close link between the complementarity of specific properties in each of the mental and physiological domains and their non-causal correlations. Section IV will show that time can be understood as a psychophysical interface between mind and matter in as much as it orders both causal chains of events and can thus be used to parametrize their correlation.

\section{The psychosomatic unity of the individual}

Brain imaging techniques provide us with evidence about the existence of neural correlates of mental processes. For example, it has been shown that visual perception is correlated with the activation of a multitude of specialized brain areas that process the different aspects of the perceived object (information about its shape, its colour, its orientation or its state of movement...). These areas are distributed along two different (dorsal and ventral) streams beginning from the primary visual area V1 of the occipital lobe, respectively reaching the posterior parietal cortex and the inferotemporal cortex and ending in the prefrontal cortex (Koch 2006). Moreover, experiences of binocular rivalry have shown that "high level" areas of the ventral stream are more specifically involved in conscious visual perception (Leopold and Logothetis 1996). Indeed, as suggested by the neurologist Damasio, the whole body would be involved in the very making of consciousness by taking into account the role played by emotions. This author explains that information about emotional reactions of the body is inserted (in the cortex) to the cognitive and motor tasks and gives then rise to "the feeling of what happens." (Damasio 1999)

In our daily life we experience the close relation between our thoughts or the emotions they give rise to and our body reaction. For example, one can observe that "negative" emotions, like fear, anger, anxiety or hostility are generally correlated to palpitation of the heart, elevation of blood presure or a disturbance of the respiratory system. On the basis of precise 
evidence $^{1}$, medical research has reported the fact that chronical stress increases vulnerability to illness by more frequently stimulating the sympathetic nervous system whose general action is to prepare the individual to "fight-or-flight" response -which gives rise to an increasing of adrenalin and cortisol production by the surrenal glands and to a disorder in the immune system. The symmetric phenomenon can also be observed since "positive" emotions like joy, pleasure or enthusiasm generally correlate with healing mechanisms (involving the activation of immune defences) via the stimulation of the parasympathetic nervous system which works for maintaning the body at rest (for example, by slackening of muscular tensions).

The holistic approach of the individual and his illness has been explored for a long time. For example, the ancient Greek physician Hippocrates conceived illness as a loss of global harmony of the body and the soul of an individual that could be restored by taking into account his "temperament" and his personal experiences. The term "psychosomatic" has been suggested by the psychiatrist Heinroth to emphasize this indivisibility of mind and body, for example by considering madness as a disease of the entire being. According to this line of thought, Freud has introduced the concept of hysterical conversion to denote the "jump in the organic", through somatic symptoms, of some "psychical excitation" (Freud 1895). Groddeck, a contemporary of Freud, has even suggested that any organic disorder would be the expression of psychical disorder (Groddek 1923). In order to explain that "each physiological process is directly or indirectly influenced by psychical stimuli" and that, consequently, "any illness would be psychosomatic", the physician and psychoanalyst Alexander, from the School of Chicago, has also suggested that all features of the human being, whether psychic or somatic, are bound together into an interdependent whole (Alexander 1950). The idea according to which psychism plays an essential role in the birth of organic illness and in the healing process has been systematically explored since the sixties by Marty and other French psychoanalysts who founded the Psychosomatic School of Paris. On the basis of clinical observations and theoretical models, these researchers have advocated a holistic approach to illness relying on the idea that the somatization process would be triggered by the absence of a sound regulation of mental excitation by the psychism.

Generally speaking, and without focusing, like in the psychoanalytic approach, on the possible "somatizations" of psychological conflicts, it has been demonstrated, in agreement with Alexander's holistic view of the individual, that the whole psychic life (being conscious

\footnotetext{
${ }^{1}$ Such evidence is, for example, reported and explored by the surgeon and psychoanalyst Thierry
} 
or not) is strongly correlated with the whole physiological processes, the metabolism, the endocrin and immunological mechanisms of the body. Illustations of these correlations, which are studied by psycho-immuno-neuro-endocrinology, have been given above when we mentioned the somatic correlates of positive and negative emotions. Another striking illustration of this radical inseparability between mental and organical processes is provided by the so-called "placebo effect", where a pharmacologically inert substance such as a sugar tablet or a saline solution can produce the same physiological effect than that of a genuine pharmacologically active substance (such as an antibiotic). This effect, which has been observed in rigorous, double blind experiments, essentially relies on the physician's belief in the treatment and the patient's faith in the physician, that is, on purely psychological factors (Janssen 2006). Reciprocally, let us mention that any change in the physiological processes can have an "effect" on psychism. For example, as reported by psychiatric studies, a change in the release process of neurotransmitters that takes place in chemical synapses (a change which can be triggered by drugs administration) correlates with an alteration of mental states.

The concept of "psychosomatic unity of the individual" refers to this thorough entanglement of the psychic and the somatic features of a same person. It suggests that these features take part of the same underlying level of reality in spite of their own specificities. According to this line of thought, the distinction between the psychic and the somatic features of the individual should thus be understood as an epistemic distinction, relying on our means of investigation: psychology (and affiliate sciences) describe the mental features of the individual whereas biology, chemistry and (somatic) medical sciences describe his physiological features. Moreover, these two excluding mental and somatic characterizations of the individual can be qualified of complementary descriptions since they have to be considered together in order to obtain a complete specification of his global state.

This situation can be illustrated by a picture drawn from the book of John Briggs and David Peat (1984) entitled "Looking Glass Universe. The Emerging Science of Wholeness." On this picture (fig. 1) one can see a fish in an aquarium and two TV cameras which are recording its movements from two different angles and transmitting the information to two separate monitors that you are watching in another room. 


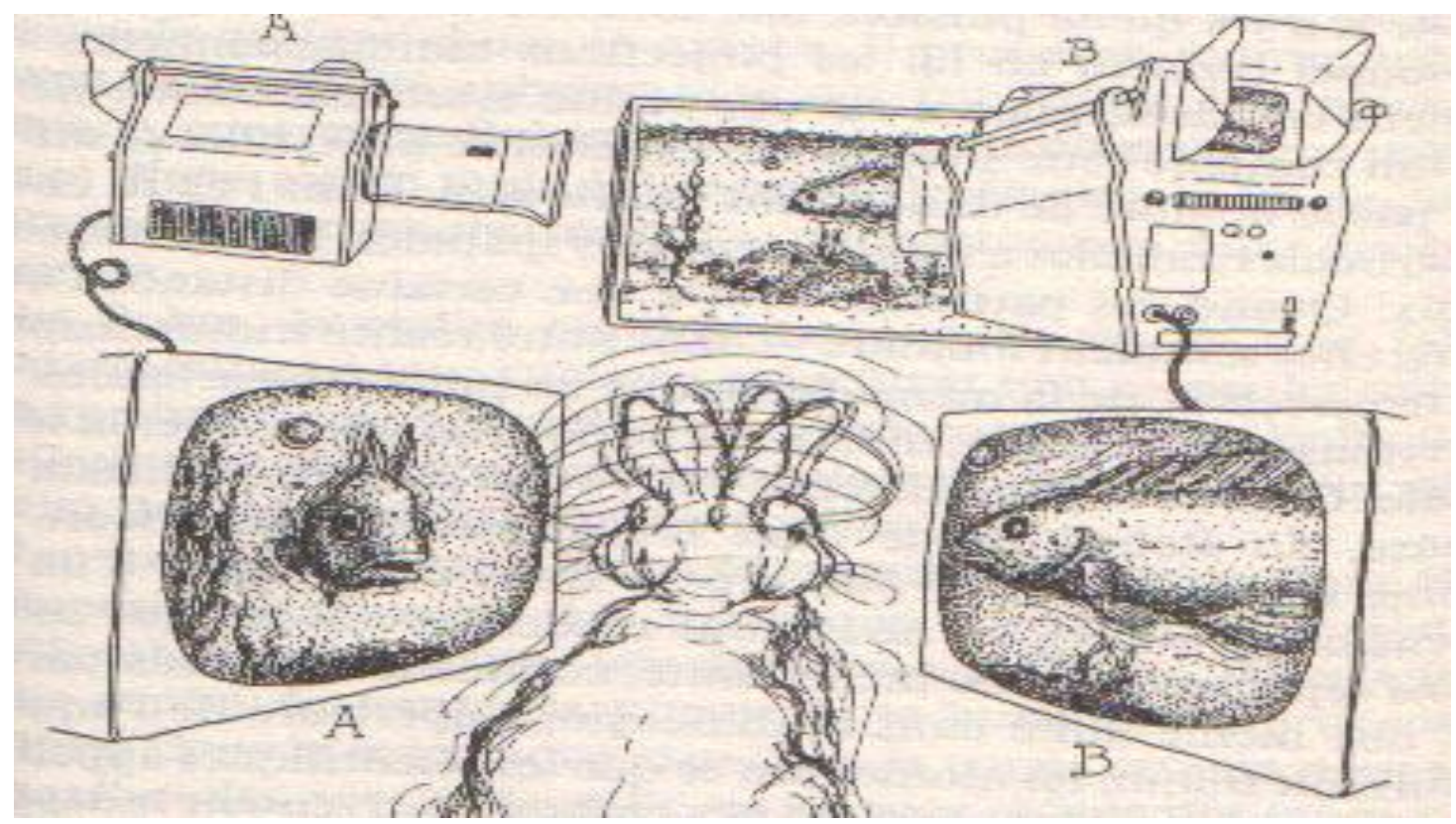

Fig. 1. There is only one fish which is observed under two different aspects. (from J. Briggs and D. Peat's book 1984)

If you are not aware of the real situation, it may seem that you are watching two different fishes which instantaneously communicate. For example, when one of them is turning, the other makes a corresponding turn and when one of them faces the front, the other one faces the side. The explanation of such correlations is of course that there is only one fish which is observed under two different, complementary aspects. In the same way, we could say that the psychic and the somatic characteristics of the individual are strongly correlated because they are complementary aspects of a same underlying level of reality, namely, the indivisible psychosomatic unity.

This illustration can be completed by a second one that more specifically focuses on the dynamical process of enfoldment-unfoldment of the mental and material aspects of the individual in a same underlying level of reality, namely the psychosomatic unity. It is the hologram metaphor which has been originally suggested by Bohm and Hiley in their implicate order theory (Bohm and Hiley 1993): a hologram is a three-dimensional photograph of an object. To make such a photograph, the interference pattern of two light beams which, respectively, arrive directly and after reflexion on the object is recorded on the photographic plate (fig. 2). When the interference pattern is illuminated with laser light, it produces a threedimensional image of the original object (fig. 3). 


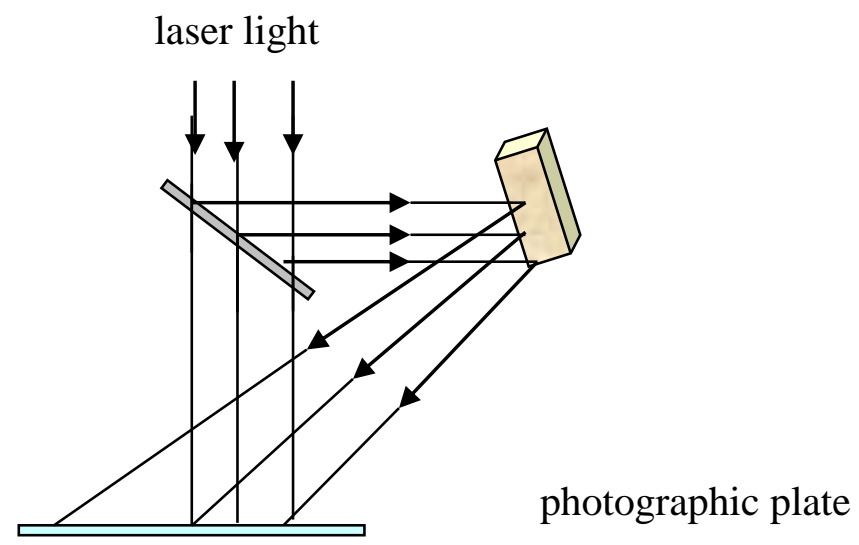

figure 2 laser light

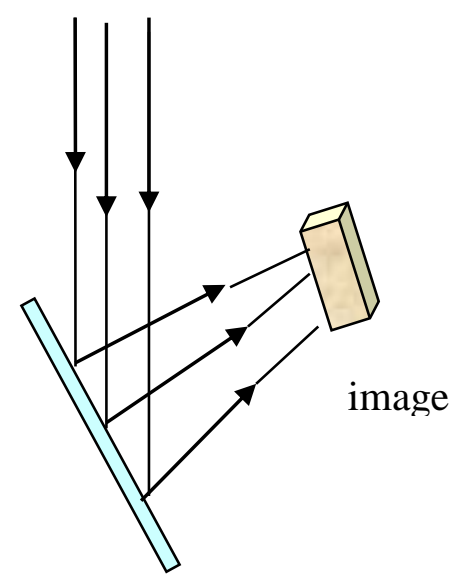

figure 3

As the two pieces of information respectively carried by the two light beams are encoded in the seemingly meaningless entangled swirls of the interference pattern and can give rise to the three-dimensional image by an appropriate action (of illuminating with laser light), one can say that the psychical and the physical aspects of the individual are enfolded in an inaccessible, underlying level of reality (namely, the psychosomatic unity) from which they co-emerge and appear as distinct by appropriate descriptions.

\section{A philosophical framework to think the psychosomatic unity.}

As shown by the previous developpements, mental and physiological processes are strongly correlated. The question of explaining the nature of this correlation has received very different responses, each of them relying on specific philosophical presuppositions about the nature of mind and matter, and about the possible primacy of one of them.

According to Cartesian philosophy, matter and mind are distinct, independent substances, respectively called "res extensa" by referring to the property of material things to be extended in space and "res cogitans", the thinking thing which is not extended in space. Their correlation, which makes our capability of having voluntary bodily motions and sensations possible, was explained by Descartes in terms of causal interaction between mind and body. This interaction was supposed to take place in the pineal gland, an organ situated at the center of the brain, between the two hemispheres. For, according to Descartes, the pineal gland is the only organ which does not exist in pair (that is, symmetrically, in the two hemispheres) and which has this priviledged position (between the two hemispheres and just above the Sylvius aqueduc which, according to Descartes, was supposed to interact with it and give rise to 
sensations). Current neurobiology has undermined Descartes' assertions about the pineal gland and its alleged role in the mind-body interaction. However, Descartes'dualism of substances has been upheld by appealing to the subjectivist interpretation of the wave function collapse suggested by von Neumann, London and Bauer or Wigner. According to this interpretation, the subject's "consciousness", "mind" or "faculty of introspection" would play an essential role in the collapse of the wave function by selecting one of the possible results of a measurement. For example, Beck and Eccles have suggested that mind could subtly change the probability distribution associated with exocytosis (the release of neurotransmitters) and, consequently, influence the information transfer between neurons -a phenomenon which would be amplified to the macroscopic level in reason of the large number of synaptic clefts of the pyramidal neurons of the cortex (Beck and Eccles 1992).

However, if it is more tenable to focus on the exocitosys phenomenon which takes place in all chemical synapses of the brain rather than on the role of some particular organ, the nature of this "interaction" between the two inhomogeneous, mental and material substances still remains unclear. If physics accounts for physical causality and psychology for mental causality, what could the laws governing psychophysical causality be ? Models of such psychophysical interaction have been provided but they are not, up to now, very convincing. For example, Walker has suggested to evaluate the action of mind on matter by asserting that the amount of information processed by consciousness in a physical (quantum) transition is equal to Shannon's statistical information associated with the probability of this transition (Walker 1975). In order to increase the maximal psychokinetic “effect", Walker's suggestion has been refined by Mattuck (1980) by assuming that mind would be capable of performing a quantum transition in several steps. However these attempts not only give rise to controversial conclusions relative to the alleged powers of mind (Alcock 2003) but, like for Beck's and Eccles' model, they rely on a subjectivist interpretation of the wave function collapse which is far to be accepted by the scientific community for its unclarity and its lack of coherence (Uzan 2011). Even more, this interpretation seems rather useless in regard to other attempts to explain the wave function collapse within the framework of quantum mechanics, for example in the decoherence approach proposed by Zurek (1981-1982) -that is, without appealing to any particular role of "consciousness" or of the "faculty of introspection" of the observer.

The question about the nature of such a psychophysical "interaction" becomes meaningless if we adopt, like in current neurobiology, a materialist position according to which all phenomena in nature could be explained by matter and by its laws -which entails, in particular, that mental processes and subjective experience could be explained from brain 
processes. However, as will be explained herafter, the materialist approach leads to the difficult question of the "explanatory gap" between the objective description of brain processes in the third person (in the language of neurobiology) and the associated subjective experience which is lived in the first person. Let us explain this point.

The materialist approach of the mind-body problem can indeed be subdivised into two options that respectively lead to two very different conceptions of scientific knowledge :

1) a reductionist approach according to which mental processes are nothing but brain processes. This conception is, for example, expressed in Minsky's claim that "Minds are simply what brains do" (Minsky 1988), or by Tononi when he defined subjective experience as "integrated information" (Tononi, 2007). It is pushed to the extreme by Dennett (1991) who contends that subjective experience would be nothing but an illusion produced by the integration of sensori-motor brain information processes and maintained by culture.

2) an emergentist approach according to which brain processes would "give rise" or "produce" mental processes and, in particular, subjective experience. According to this direction of research, psychic life could be thought of as a by-product or an epiphenomenon of brain processes, without any function or any possible "effect" on body (Ribot 1899) -like the noise produced by a car is useless for its functioning. However, as emphasized by Flanagan (1994), the very possibility to realize experiments ${ }^{2}$ that detect the moment a subject becomes conscious of perceiving a stimulus and decides or not to consciously react already seems to undermine the epiphenomenalism doctrine. Moreover, such a version of emergentism can easily be criticized by merely mentioning the fact that, as reported in section I, subjective experience can give rise to important changes in body and should therefore be endowed with some autonomy.

A more interesting version of emergentism that takes into account the autonomy of subjective experience has been upheld. According to this position which has been, for example, explored by Searle (1997), by Sperry (1983) and, more recently, by Lestienne (in this volume), the mental is understood as an emergent, higher-level property of the brain which is different from its physical properties and governed by its own laws that transcend those governing the brain processes. Searle upholds the thesis of "biological naturalism" of consciousness: on the one hand, consciousness would be a biological phenomenon "caused" by brain processes of the most evolved animals; on the other hand, this phenomenon would be "natural" like digestion, photosynthesis or mitosis. According to this emergentist position,

\footnotetext{
2 Any experiment about visual consciousness can be mentioned (Koch 2006).
} 
mental phenomena and, in particular, consciousness could not be exclusively explained from the individual properties of brain constituents or even from their combination but should be understood as global properties of the brain whose emergence requires a minimal level of complexity (acquired in evolution). Moreover, as clearly suggested by Sperry, the subjective properties are supposed, in turn, to be capable of exerting "control over the biophysical and chemical activities at subordinate levels" -which would endow some autonomy to consciousness without upholding a dualistic position.

However, if the latter approach clearly avoids reductionism by asserting that the mental should be understood as a new, emergent property of the brain, it is faced with the question of the explanatory gap. As complex as they could be, how neural processes, which are governed by material (biophysical or biochemical) laws identical for all brains, give rise (or "cause") subjective experience, that is, a sensible quality which can only be experimented in the first person? Subjective experience can be defined by "what it is like" to perform a cognitive act, like that of perceiving a red flower, or to perform an action, like that of raising the arm, and it thus refers to the content of consciousness. However, as explained by Noë and Thomson (2004), this content is relative to an ensemble of personal characteristics, like the subject's own sensibility, his beliefs, his desires and his own rationality, whereas physiological processes are of sub-personal nature and do not consequently rely on personal characteristics. How could a description of sub-personal, physiological processes in the third person explain the private experience of a particular subject? Assuming the primacy of matter can certainly allow a description of neural correlates of subjective experience; however, can such an objective description account for the private content of subjective experience?

Conversely, let us note that the "symmetric" idealist approach according to which mind (or soul) would be primary leads to analogous questions. If mind is considered as primary, matter can either be reduced to mind or thought of as an emergent property of mind. In occidental philosophy, this kind of monism has been supported by Berkeley. According to his "immaterialist" doctrine, sensible objects have no existence out of the mind of those who perceive them or, in other words, material processes are nothing but what mind perceives. Beside this reductionist view, one can uphold the proposition according to which matter is a manifestation of mind or that it would "emerge" from mind. However, in this case (which, to my knowledge, has not been explored in detail) we are faced with the question to explain how private, subjective experience could give rise to material processes governed by objective laws where all personnal references have been ruled out -a "symmetric" explanatory gap question. 
To sum up, we can say that we are faced with two difficult questions, depending on the philosophical position we adopt :

1) the question of explaining the mysterious "interaction" between mind and matter if they are considered as distinct substances, or

2) the question of the explanatory gap if we assume that one (and only one) of these two substances (mind or matter) exists.

However, a third philosophical position seems to be susceptible of disolving these two questions by asserting that both mind and matter are emergent properties of a deeper level of reality, a substance which is neither mental nor physical. This position, called neutral monism, has been developped by Spinoza very rigorously (in an axiomatic-like presentation) in the Ethics (1677, part I and II) and has been later re-interpreted in purely epistemic terms by Russell, by James and by Mach on slightly different grounds. Neutral monism has also been explored by Jung and Pauli from the point of view of the deep psychoanalysis (theory of archetypes) and, more recently, within the framework of quantum theory, by Bohm and Hiley and by Atmanspacher and Primas. Let us briefly present this philosophical system wherein the notion of psychosomatic unity can be fruitfully explored.

According to Spinoza, there exists only one infinite, eternal, substance (also called God or Nature) which is neither mind nor matter. Everything in reality is considered as a mode or a modification of this "neutral" substance. Mind and matter are understood as two "attributes" of this single substance, that is, as two intellegible aspects of it, as what we can perceive of it. In Spinoza's system, one can thus already find the idea of an epistemic distinction between the two, mental and physical aspects of the substance. This idea has been taken and extensively developed by Russell in his "Analysis of Mind" (1921). This author asserts that we apprehend the neutral substance with different "logical constructions" : physics is used to apprehend its material aspect and psychology its mental aspect.

In the Ethics, one can also find the idea of a psychophysical parallelism regarding the two, mental and physical, aspects of the substance (and not regarding two distinct substances, like in Leibniz's parallelism) since, for Spinoza, "the order and connection of ideas are the same as those of things", which means that the laws which respectively govern the two, mental and physical processes are synchronized. This idea, which will prove very fruitful for characterizing their relationship in terms of time entanglement (see section IV), has been more recently explored by Pauli who has suggested that the mental and the material fields are governed by common ordering principles (Pauli 1994). Moreover, following Yovel's analysis (Yovel 1989, chap. 6), one can assert that, in order to defend his radical monism, Spinoza has 
also upheld the idea of complementarity of mental and material descriptions of the neutral substance, an idea later explored by Pauli who has asserted that mind and matter should be understood as "complementary aspects of the same holistic and indivisible reality" by explicitly referring to the concept of complementarity in quantum theory.

It consequently seems that neutral monism is very suitable for exploring the notion of psychosomatic unity since it leads to the essential features of the mind-body connection mentioned in section I:

1) the idea of an epistemic distinction of mental and somatic features of the individual, which can thus be understood as complementary aspects of the psychosomatic unity

2) the idea of entanglement, and more precisely, that of synchronisation of mental and physiological processes ("parallelism" of aspects), which will be explored in detail in the next sections.

\section{Complementarity and Entanglement beyond Physics.}

As shown by the previous analysis, the psychosomatic phenomenon esentially relies on the properties of complementarity and entanglement of mind and body. However, these two properties have initially been defined in quantum physics to characterize quantum phenomena. We consequently have to explore the possibility of rigorously applying these notions beyond the strict framework of quantum physics.

In quantum physics, two physical quantites A and B are said to be "complementary" if the corresponding observables ${ }^{3}$ which represent them mathematically do not commute :

$$
[\mathrm{A}, \mathrm{B}]=\mathrm{df} A \mathrm{AB}-\mathrm{BA} \neq 0 .
$$

For example, the position observable $\mathrm{X}$ and the momentum (in the $\mathrm{X}$-direction) observable $\mathrm{P}_{\mathrm{X}}$ do not commute :

$$
\left[X, P_{X}\right]=i \hbar I,
$$

their degree of non-commutativity being evaluated by $\hbar$, the Planck constant divided by $2 \pi$.

Let us have a look at the possible interpretations of the notion of complementarity in quantum physics in order to better understand its possible generalizations. The relation (1) literally means that the sequence in which $A$ and $B$ are measured is not indifferent. If $\mathrm{A}$ and $\mathrm{B}$ are complementary and that B is measured first, the measurement of A performed after that of $\mathrm{B}$ disturbs the state of the system and generally erases the previous value of $\mathrm{B}$, as can be

3 Observables are linear auto-adjoint operators acting on the space of representation (Hilbert space) relative to the phenomenon under study. 
shown by a second measurement of B. This can be shown by using three Stern-Gerlach devices that successively measure the spin of a particle according to two different directions (respectively, for A and for B) -which defines two complementary observables (CohenTannoudji and al. 1997, chap. IV). Even more generally, in as much as a measurement can be understood (and is mathematically represented) as the action of an operator on the state of the system under study, one can interpret the notion of complementarity of observables in terms of complementarity of actions performed on this system: the action associated to one of the two observables is antagonist to (and erases) the action of the other, yet both actions are required in order to fully specify its state.

The notion of "entanglement" was originally introduced by Schrödinger to characterize the impossibility of describing by two independent states two quantum systems which interacted in their common past (Schrödinger 1935, p. 555). Entanglement is mathematically expressed by the fact that the state of the system $\mathrm{S}$ composed of the two subsystems $\mathrm{S}_{\mathrm{I}}$ and $\mathrm{S}_{\mathrm{II}}$ can only be written as a non-factorable state of the form :

$$
\left.\left.\left|\Phi>=\mathrm{c}_{1}\right| \varphi_{1}^{\mathrm{I}}\right\rangle\left|\varphi_{1}^{\mathrm{II}_{1}}\right\rangle+\mathrm{c}_{2}\left|\varphi_{2} \mathrm{I}_{2}\right| \varphi^{\mathrm{II}}{ }_{2}\right\rangle+\ldots \ldots,
$$

where the products $\left|\varphi_{i}^{I_{i}}\right\rangle\left|\varphi_{i}^{I I}\right\rangle$ are the possible global states of $S$-which means that $|\Phi\rangle$ cannot be written as the product of a single state of $S_{I}$ and a single state of $S_{\text {III }}$ In this case, correlations exist between $S_{I}$ and $S_{\text {II }}$ even if $S_{I}$ and $S_{I I}$ are causally separated ${ }^{4}$ since if $S_{I}$ is found in the state $\left|\varphi_{\mathrm{i}}^{\mathrm{I}}\right\rangle, \mathrm{S}_{\text {II }}$ will be necessary in the corresponding (or "relative") state $\left|\varphi^{I_{i}}\right\rangle$. This phenomenon has been successfully tested in Aspect's experiments (Aspect 1982) by measuring the polarization of two photons prepared in such an entangled state:

$$
|\Phi>=1 / \sqrt{ } 2(|\uparrow>| \uparrow\rangle+| \rightarrow>\mid \rightarrow>),
$$

where $\mid \uparrow>$ and $\mid \rightarrow>$ respectively denote the vertical and horizontal polarization states of the photons (fig. 4). These photons are emitted in two opposite directions and their polarization is measured according to different angles by the appropriate devices D1 and D2 :

\footnotetext{
${ }^{4}$ This means that there is no possible causal interaction between these two sub-systems.
} 


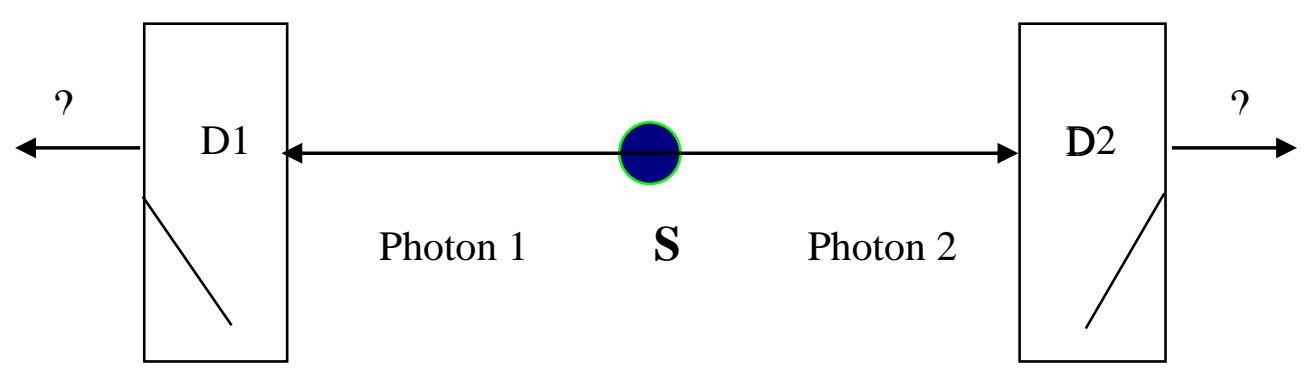

figure 4: a schematic diagram of Aspect's experiment

The outcomes of measurements performed on a big number of such "twin photons" show that their polarizations are strongly correlated, in agreement with the prediction of quantum theory. Yet, the experiment is performed in such a way that any possible exchange of information between them or between the different parts of the experimental device has been ruled out ${ }^{5}$. Moreover, what is essential, the particles did not actually possess before the observation the properties (polarizations) measured by the devices D1 and D2. This fundamental point has been established by the violation of some statistical conditions known as Bell's inequalities ${ }^{6}$. These correlations which cannot be explained by classical causation are thus qualified of "non-local" or "non-causal".

As mentioned above, the notions of complementarity and entanglement can be applied (or even re-defined) beyond their original context, beyond physics and even beyond the material realm. Bohr has suggested to use the notion of complementarity in any area of reality, to designate the relationship between excluding descriptions which have to be considered together in order to obtain a complete information about a phenomenon. It is the case for the well-known example of the wave-corpuscle complementary aspects of light: light can be modelled as being composed of corpuscles (photons) by observing their impact on a screen, but light can also be described as a wave since it gives rise to wave phenomena, as is the case for the interference pattern in Young's experiment (see below). Beyond physics, Bohr used it to characterize the relationship between the mechanistic and teleological aspects of life development which respectively involve causality and finality principles. In psychology, he

\footnotetext{
${ }^{5}$ In the words of relativity, the measurement-events of these properties (spin) are said to be spatially separated.

${ }^{6}$ Bell's inequalities are conditions that must be held for any statistics bearing on a population whose individuals are supposed to carry intrinsically their properties. The first formulation of such conditions was established by the physicist John Bell (1964) but it is the more suitable formulation provided by Clauser, Horne, Shimony and Holt (1969) which is tested here.
} 
used this notion to characterize the relationship between the mental attitude of spectator and that of actor which exclude each other but are both necessary in life.

The notion of complementarity of descriptions can be rigorously characterized in algebraic terms. A "description" can be mathematically defined as an algebra of propositions, that is, as a set of propositions susceptible to describe the phenomenon under study and of logical connectors (and, or, not,..) governed by a system of logical rules. A description governed by the laws of classical logic (like the law of excluded middle or the distributivity of the "and" over the "or") defines what is called a Boolean algebra. The notion of complementarity of descriptions can now be defined as follows (Primas 2010) :

Two descriptions are "complementary" if they cannot be embedded into a single Boolean algebra.

In other words, two complementary descriptions cannot be combined in order to form a single description governed by classical logic. This definition can be illustrated by the wavecorpuscle complementarity of light mentioned above. In Young's double slit experiment, the complementary descriptions of light are, respectively, relative to different experimental contexts: when both slits A and B are open (fig. 5), one can observe an interference pattern by detecting the position of the particles only on the screen, which gives rise to a wave-like description expressed by a first experimental language $\mathrm{L}_{\mathrm{AB}}$.

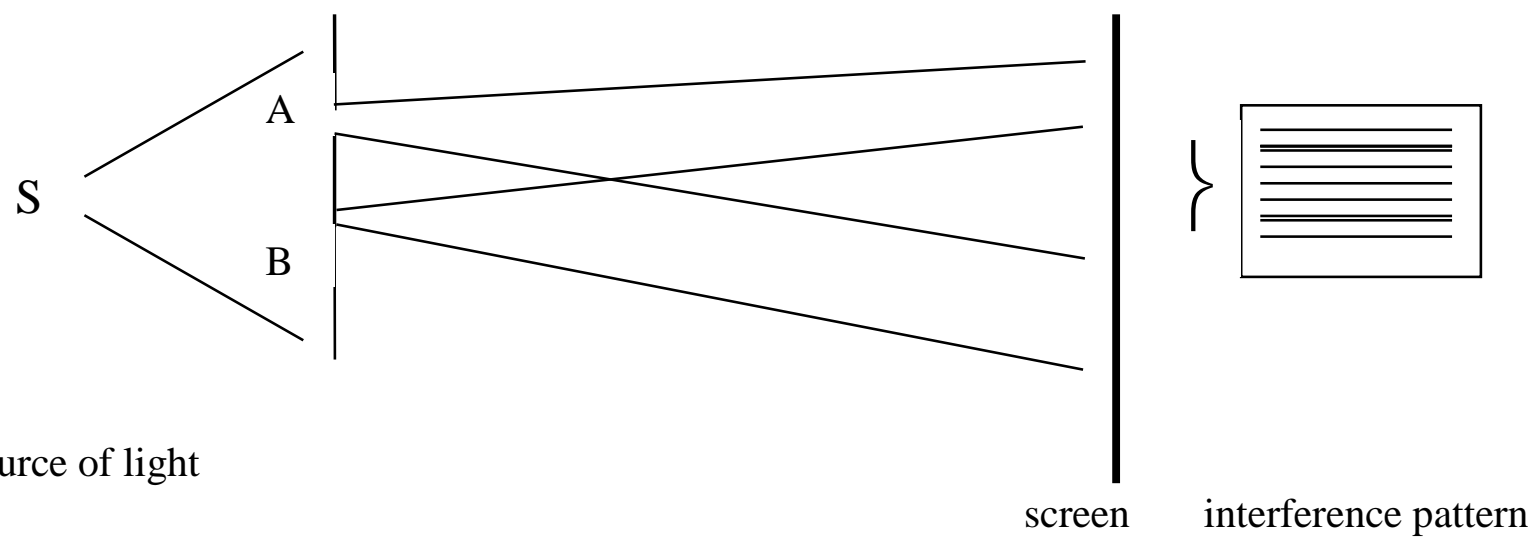

fig. 5: Young's double slits experiment

However, one can also determine which slit the particles pass through by placing counters at the slits. In this case, if we can know with certainty which slit the photon passed through, the interference pattern disappears, which means that we can only observe the corpuscular 
aspect of light ${ }^{7}$. In fact, three different similar experiments can be performed depending on which slit is open (only A, only B or A and B) -which defines three corpuscular descriptions $\mathrm{L}_{\mathrm{A}}, \mathrm{L}_{\mathrm{B}}$ and $\left(\mathrm{L}_{\mathrm{A}} \vee \mathrm{L}_{\mathrm{B}}\right)$ which, respectively, account that the photon passed through $\mathrm{A}$, through B or through one the two slits (Bitbol 1996, § 1.2.10). It can be shown that the corpuscular descriptions $\mathrm{L}_{\mathrm{A}}, \mathrm{L}_{\mathrm{B}}$ and $\left(\mathrm{L}_{\mathrm{A}} \mathrm{v} \mathrm{L}_{\mathrm{B}}\right)$ can generate a Boolean algebra but that the wave-like description $\mathrm{L}_{\mathrm{AB}}$ and the corpuscular descriptions cannot be embedded into a single global Boolean algebra (Bitbol 1996, annexe 1) -hence the complementarity of corpuscular and wave descriptions.

The notion of complementarity of observables, which is more specifically used in quantum physics, can also be generalized. To implement this idea in a precise way, one has to mention Atmanspacher's, Römer's and Walach's proposition (2002) to work out a generalized interpretation of quantum theory where all references to the physical world have been relaxed. In such an interpretation, there is no Planck's constant (which characterizes the quantum domain for physical phenomena), no a priori probabilistic interpretation (namely, Born's rule, which calculates the probability distribution of outcomes of measurements of physical quantities) and no Schrödinger evolution (evolution law of physical systems). In this generalized quantum theory, a "system" is defined as any part of reality which is an object of attention and investigation and an "observable" can denote any meaningful property which can be measured or observed in a reproducible way ${ }^{8}$. This general application of quantum theory had been implicitly assumed by Atmanspacher and Scheingraber (1987) in order to show the complementarity of the Hamiltonian dynamics and of the informational dynamics for chaotic systems in classical physics. The complementarity of these two modes of dynamical descriptions is expressed by the non-commutativity relation:

$$
\mathrm{i}[\mathrm{L}, \mathrm{M}]=\mathrm{K} \mathrm{I}
$$

where $\mathrm{L}$ is the Liouville operator, which governs the Hamiltonian evolution, $\mathrm{M}$ an operator which computes the amount of information that can be gained by measuring an ensemble of key quantities on this system and $\mathrm{K}$ is the Kolmogorov-Sinaï entropy (a positive invariant for

\footnotetext{
${ }^{7}$ However, if we do not know with certainty which slit the photon passed through, the visibility of interference at the screen is only reduced. In this intermediate case, both aspects of light co-ecist. For the sake of simplicity, we only consider here the extreme cases.

${ }^{8}$ These authors also suggest to weaken the algebraic structure of standard quantum theory. However, such a weakening of the standard formalism of quantum theory is not appropriate for our purpose and will not be retained.
} 
chaotic systems $)^{9}$. This non-commutativity relation implements the idea according to which the accuracy of prediction decreases with increasing time.

Beyond physics, in the field of psychology of perception, Atmanspacher, Filk and Römer (2009) have recently applied the notion of complementarity to the phenomenon of bistable perception, considering the Necker cube. This two-dimensional representation of a cube can be seen from above (front face up and at left) or from below (front face down and at right), and can thus be modelled as a quantum system oscillating between two non-stationary states (a) and (b) and submitted to observation :

(a)

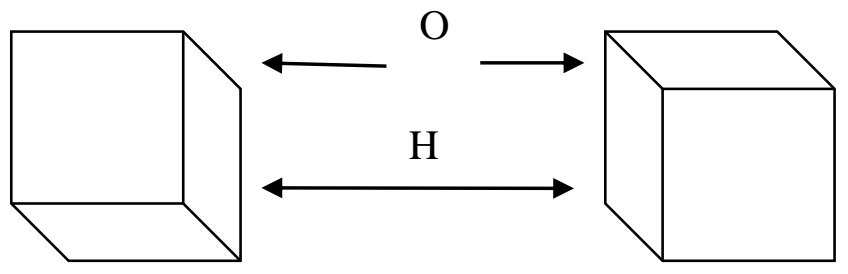

figure 6: the two possible perceptions of a Necker cube

These authors have established a relationship of complementarity between the continuous, non-disturbed Hamiltonian-like dynamics $\mathrm{H}$ (which is an oscillation between the two possible perceptions of the cube (a) and (b)) and the abrupt change of perception governed by an observation operator $\mathrm{O}$ whose eigenstates are (a) and (b).

Still more generally, Römer (2006) has mentioned the complementarity relationship between substantial and "processual" observables in any domain of reality: substance observables refer to timeless features of a system, like the possible states in which it can be found, alluding thus to Parmenidean philosophy, whereas process observables are relative to its change, to the transition between these states and refer to Heraclitean philosophy. A wellknown consequence of such a complementarity between substance and process observables is the "quantum Zeno effect" which we already met indeed in the model of bistable perception (Necker cube) previouly presented: the Hamiltonian evolution of a quantum system (represented by a process observable) can be slowed -and even frozen - by observing it frequently (that is, by a frequent application of a substance observable on its state).

9 Note that contrary to the case of non-commutativity of observables in quantum mechanics, which always involves the Planck constant, the commutator $[\mathrm{L}, \mathrm{M}]$ is not universally determined here since $\mathrm{K}$ is system- and parameter-dependent. 
The notion of entanglement can also be generalized beyond physics. As is the case for that of complementarity, such a generalization relies on the possibility of applying the quantum formalism beyond the strict field of quantum (physical) phenomena and even beyond the material realm. As a matter of fact, Baaquie and Martin (2005) have proposed a quantum model of the psyche where the latter is represented as an entanglement of elementary "ideas" weighted by a probability law. A representation of the psychosomatic entanglement involves the representation of specific mental and somatic features of the individual. This can be done by referring to an important link which can be established between the complementarity of local observables, defined on different parts of a generalized "system", and their entanglement. This link has been shown by Landeau (1987) within the $C^{*}$ algebra of observables that underlies the standard quantum theory. Landeau's theorem is a generalization of Bell's inequalities which have been tested in Aspect's experiment in order to show the nonlocality of quantum correlations (see above).

Let us consider two couples of observables A, B and A', B' respectively measured on two causaly separated regions, called $\mathrm{S}_{\mathrm{I}}$ and $\mathrm{S}_{\mathrm{II}}$, of a system $\mathrm{S}$ (fig. 7). Landeau's theorem sets out, in the classical case (where all observables commute) and in the quantum case (of non commutative observables), the conditions fulfilled by the correlation factor $\mathrm{R}$, defined as:

$$
\mathrm{R}=\mathrm{S}\left(\mathrm{a} \mathrm{a}^{\prime}\right)+\mathrm{S}\left(\mathrm{a} \mathrm{b}^{\prime}\right)+\mathrm{S}\left(\mathrm{b} \mathrm{a}^{\prime}\right)-\mathrm{S}\left(\mathrm{b} \mathrm{b}^{\prime}\right),
$$

where $\mathrm{S}$ ( $\mathrm{a}$ a') is the expectation value of the product of the results of measurement of $\mathrm{A}$ and $\mathrm{A}^{\prime}$, and we have a similar definition for the other terms.

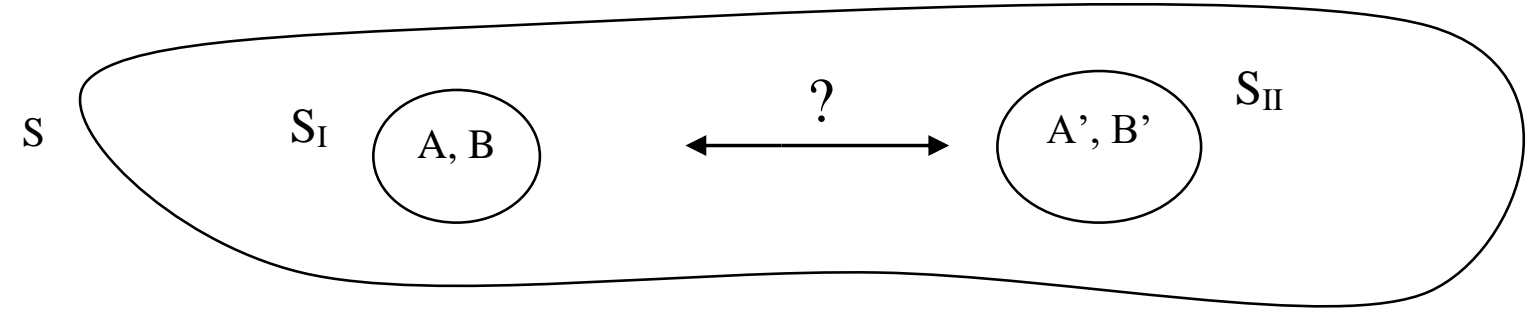

figure 7: how correlated are $S_{\text {I }}$ and $S_{\text {II }}$ ?

As expected, this theorem reduces to the usual Bell's theorem in the classical case of commutative observables:

$$
[\mathrm{A}, \mathrm{B}]=0 \text { or }\left[\mathrm{A}^{\prime}, \mathrm{B}^{\prime}\right]=0 \quad \Rightarrow \quad-2 \leq \mathrm{R} \leq+2 \text {, }
$$

which characterizes (classical) local correlations. However, in the quantum case of noncommutative observables, Landeau's theorem provides us with a very powerful means to 
capture the concept of generalized entanglement and its intimate link with that of complementarity. This theorem shows that the possibility of defining non commutative properties in each of the two parts of a system is a sufficient condition for the existence of non local correlations between these two subsystems (which is expressed by the fact that $|\mathrm{R}|$ is bigger than 2, the classical limit):

$$
[\mathrm{A}, \mathrm{B}] \neq 0 \text { and }\left[\mathrm{A}^{\prime}, \mathrm{B}^{\prime}\right] \neq 0 \Rightarrow-2 \sqrt{ } 2 \leq \mathrm{R} \leq+2 \sqrt{2}
$$

This result, which is very general since its derivation does not a priori refer to physical properties of the considered "systems" ${ }^{\text {, }}$ can thus be used as follows for modelling the psychosomatic phenomenon:

(i) the subsytems $S_{I}$ and $S_{\text {II }}$ can be thought of as, respectively, the mental and the somatic representations of the same indivisible psychosomatic unity

(ii) specific, mental and somatic, features of the individual can be represented in a precise manner by generalized observables defined, respectively, on these subsystems, and

(iii) the psychophysical entanglement that characterizes the very notion of individual can be explained and explored from the complementarity of some of these observables in both domains.

According to this line of thought, simple models of the psychosomatic phenomenon can be provided. For example, the somatic aspects can be characterized by the actions of specific hormones on the body, whereas mental aspects are described by psychic features or "temperaments" according to a generally accepted psychic typology. As is well known, hormones actions can be complementary in as much as they exclude each other by performing opposite tasks, and yet are both necessary for body equilibrium, namely for maintaining minimal fluctuation in physiological variables (like blood pressure, blood volume or blood sugar concentration). It is the case for the couple of hormones insulin/glucagon which have opposite effects on blood glucose concentration: the latter tends to increase it whereas the former tends to lower it. Another well-known example is the couple of complementary hormones estrogen/progesterone which are involved in the process of periodic menstruation in women: these hormones have antagonist actions since they have opposite effects in the

10 Beside the use of the algebraic formulation of quantum theory, the derivation of this result essentially relies on Schwartz inequality for expectation values, which reads as follows:

$|\mathrm{E}(\mathrm{X})|^{2} \leq \mathrm{E}\left(\mathrm{X}^{2}\right)$, where $\mathrm{X}$ is a random variable. 
regulation of uterine peristalsis and are both required to ensure a delicate and vital balance. This complementarity of hormone actions can define non-commutative operators acting on the space of possible body states ${ }^{11}$.

Similarly, in the mental field, one can find couples of complementary patterns. For example, as emphasized by Bohr, passive and active attitudes are complementary, which means that they exclude each other (we cannot at the same moment act and passively observe, these attitudes being alternative) but are both necessary in everyday life. The complementarity of cold and logical reasoning and emotional reaction can also be mentioned since these two modes of mental functioning cannot co-exist at the same time but are both required in life. Or, in the process of adaptation, let us note the complementarity of assimilation and accommodation mental phases, which are distinct and exclusive but are both required to ensure a continuous dynamic of 'equilibration' (Piaget 1975). These complementary mental processes also define non-commutative operators acting on the space of mental states.

Considering now Landeau's theorem (whose derivation does not appeal to any particular domain of reality), one can conclude that the existence of such complementary couples of properties in both the mental and the physiological domain is sufficient to explain psychosomatic entanglement -which means that causal interactions are not required. Within the framework of generalized quantum theory, a precise modelling of the psychosomatic phenomenon can thus be provided as follows:

(1) we first define a space of psychosomatic states $\mathrm{H}$ (namely, a Hilbert space) as a tensor product of a space of body states $M$ defined from a finite collection of mutually exclusive (complementary) somatic properties -for example, by considering the physiological actions of the main hormones-, and a space $\mathrm{N}$ spanned by a basis of the corresponding, exclusive mental states -namely, by referring to the known psychological characteristics of these hormone actions.

(2) the psychosomatic state of an individual is represented as an entangled state of $\mathbf{H}$, that is, as a sum of products of the different possible, corresponding mental and physiological states

(3) the (epistemic) co-emergence of the specific mental and somatic features encoded in the global psychosomatic state is mathematically expressed by projections onto their respective space; moreover,

\footnotetext{
${ }^{11}$ See below for a definition of this space.
} 
(4) one can mathematically represent the evolution of such entangled psychosomatic states by defining evolution operators that account for biological and psychological rhythms and for the main interactions with the environment (Uzan 2011).

\section{Time entanglement of mental and physiological processes.}

This final section explores the role of time in the psychosomatic phenomenon. It is shown that time can be understood as a psychophysical interface in as much as it can be used to parametrize both causal chains of mental and physical events.

As shown by the long-standing discussions about the nature of time, and as emphasized by Mc Taggart (1908), two concepts of time seem to be radically opposed. Physical time parametrizes material processes and its key features can be derived from theoretical physics: (i) the invariance of the fundamental laws of physics by time translation entails that the notion of "now" to which events can be reported is missing, (ii) their invariance by time reversal prevents a representation of irreversibility of phenomena (iii) no becoming can be implemented in the block-universe conception of relativity -which is expressed, for example, by Weyl's famous sentence: "the objective world simply is, it does not happen", (iv) according to this concept of time, events are reported by tenseless sentences that order them by the relations "earlier than", "simultaneous with" and "later than" on a time axis which is set for eternity -which defines Mc Taggart's B-series.

By contrast, mental time is relative to our inner experience of time since a sequence of mental events is defined for a particular subject. According to this line of thought, Augustine upheld the idea that time exists only in the human mind's apprehension of reality -and not in reality itself-, whereas Bergson opposed his concept of "creative duration", which directly refers to our intuitive perception of time, to that of physical time. Mental time refers to a consciousness-related "now" and seems to irreversibly flow from our "past" to our "future". Mental events are thus reported by tensed concepts (Mc Taggart's A-series) since they refer to our subjective experience of time.

Is one of these two concepts of time (A-time and B-time) more fundamental than the other? How are they related? 


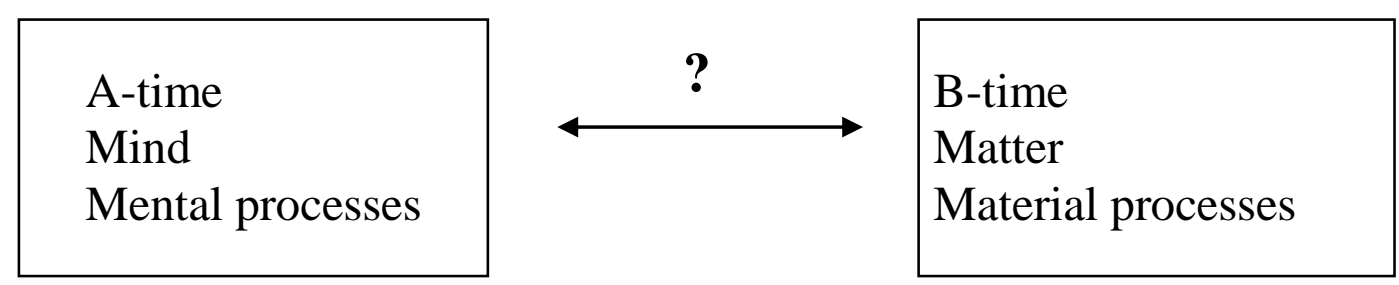

The connection between A-time and B-time relies on that between mental and material processes. According to the neutral monism philosophy we have chosen to explore here, mind and matter are complementary aspects of an underlying "neutral" stuff. They co-emerge from this indivisible level of reality and their entanglement, expressed in terms of (EPR-like) noncausal correlations, can be understood as remnant of their common origin (see sections II and III). Following this line of thought, one can characterize the relationship between A-time and B-time as follows:

(1) A-time and B-time are complementary representations of time: they exclude each other, as mentioned by philosophers, yet they are both required to describe the world (i.e. physical and mental phenomena). Each of these concepts of time has its domain of validity and, as stressed by Primas (2003), it is not very fruitful to ask which of them is the "correct" representation of time.

(2) the entanglement between mental and material processes can be expressed in terms of entanglement between A-series and B-series and, consequently, they can be parametrized by time which takes part to both series -hence the idea of time-entanglement between mental and material aspects of the psychosomatic unity. The concept of time-entanglement echoes with Jung's notion of "synchronicity" that refers to the existence of significant coincidences between inner (mental) events and outer events (Jung 1988). More specifically, it agrees with Meier's idea according to which psychosomatic phenomena could be understood as synchronistic phenomena (Meier 1988).

These conclusions, which can be schematized by the following diagram: 


\section{body, material processes}

A-time

time entanglement

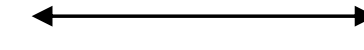

\section{mind, mental processes}

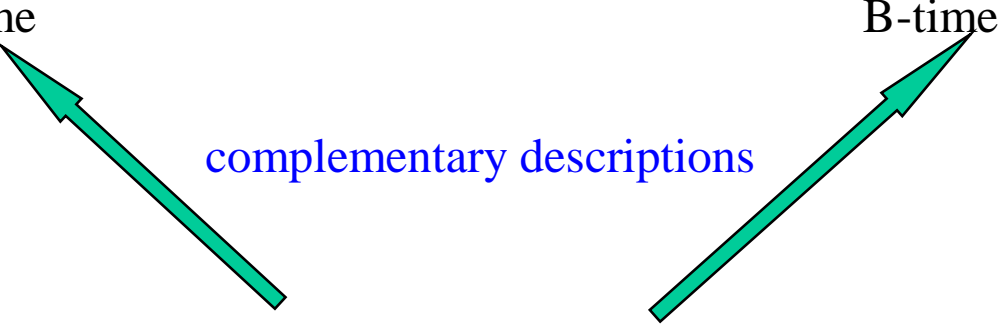

psychosomatic unity

are supported by Primas's rigorous developments (Primas 2003 and 2010) within the framework of (generalized) standard quantum theory.

According to Primas' approach, a state of the psychophysical unity is defined as a maximally entangled state ${ }^{12}$ of the Hilbert tensor product space

$$
\mathrm{H}=\mathrm{N} \otimes \mathrm{M},
$$

where $\mathrm{N}$ and $\mathrm{M}$ refer, respectively, to the mental and material domains. The symmetry of the neutral, psychophysical level is represented by the unitary one-parameter group of automorphisms $\left\{\mathrm{e}^{-2 \pi} \mathrm{i} \tau \mathrm{G} / \tau \in \mathrm{R}\right\}$ generated by the self-adjoint operator $\mathrm{G}$. The (epistemic) breaking of symmetry of the psychophysical unity gives rise to the following expansion :

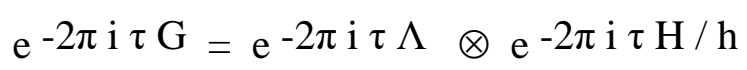

where $\mathrm{H}$ is the Hamiltonian operator, the generator of the unitary group of evolution operators of the material domain $\mathrm{M}$, and $\Lambda$ is the generator of the evolution in the psychical domain $\mathrm{N}$.

The mental domain $\mathrm{N}$ is modelled as an abstract memory whose growth defines a chronological order :

$$
\tau<\tau^{\prime} \text { if and only if } \mathrm{N}_{\tau} \subset \mathrm{N}_{\tau}{ }^{\prime},
$$

which means that the "now" is specified by a memory content and is thus unique in as much as it is experienced differently by each individual and for each of his memory configuration. The lack of time-translation and time-reversal invariance of mental time is a consequence of this memory-dependent definition of chronological order. One can say, in agreement with Augustine's philosophy of time (Confession, book XI, chap. XXVI to XXVIII), that the concepts of "nowness", "pastness", "futureness" and that of "flow of time" originate in the mental domain. 
Within this mathematical framework, Primas shows that the maximally entangled psychophysical state, which is invariant under the action of the unitary group $\left\{\mathrm{e}^{-2 \pi} \mathrm{i} \tau \mathrm{G} / \tau \in \mathrm{R}\right\}$, can be written as :

$$
\Xi=\int_{\mathrm{R}}|\tau\rangle \otimes \mathrm{e}^{-2 \pi \mathrm{i} \tau \mathrm{H} / \mathrm{h}} \Psi \mathrm{d} \tau,
$$

where $\Psi$ is a reference vector of $M$ and $|\tau\rangle$ is the state of $N$, which can be labelled by a value of the time parameter $\tau$. This means that each possible state of the mental domain $\mathrm{N}$ is associated with a specific state of the material domain M (its "relative state"). The notion of time-entanglement of mind and matter is mathematically expressed by the fact that the noncausal correlations between mental and material processes are parametrized by time, which thus appears as their common ordering principle.

\section{Conclusion}

Quantum theory provides us with suitable tools to model the psychosomatic unity of the individual : mental and physiological processes can be modelled as co-emergent, complementary aspects of the psychosomatic unity whose entanglement can be understood in terms of non-causal correlations. Moreover, such a quantum representation relies on a concept of time as psychophysical interface -neither physical, as a feature of independent reality, nor mental, as exclusively relying on subjective experience-, a concept which can prove very fruitful to explore the psychosomatic field.

\footnotetext{
${ }^{12}$ A quantum state is maximally entangled if its coefficients are equal.
} 


\section{References}

Alcock J. 2003. "Give the null hypothesis a chance.” Journal of Consciousness Studies, 10 : $29-50$.

Alexander F. 1950. Psychosomatic Medicine: Its principles and Applications, New York: Norton.

Aspect A. and al. 1982. "Experimental Realization of Einstein-Podolsky-Rosen-Bohm Gedankenexperiment: A New Violation of Bell's Inequalities”, Phys. Rev. Lett. 49: 91.

Atmanspacher H, Römer H, Walach H 2002. "Weak quantum theory: complementarity and entanglement in physics and beyond", Found Phys., 32 :379-406.

Atmanspacher H, Filk T, Römer H 2009. "Complementarity in bistable perception", in Recasting reality: Wolfgang Pauli's philosophical ideas and contemporary science. Atmanspacher H, Primas H eds . Berlin \& New York: Springer. 135-150.

Atmanspacher H. and Scheingraber H. 1987. "A fundamental link between system theory and statistical mechanics". Found. Phys. 17 : 939-963.

Augustine : Confessions, translation Chadwick Henry, Oxford: Oxford University Press.

Baaquie B. E. and Martin F. 2005. “Quantum Psyche”, NeuroQuantology 1 : 7-42.

Beck F. and Eccles J. 1992. "Quantum aspects of brain activity and the role of consciousness", Proceedings of the National Academy of Sciences of the USA, 89 : $11357-11361$.

Bell, John 1964. “On the Einstein Podolsky Rosen Paradox”, Physics 1 : 195-200.

Bitbol M. 1996. Mécanique quantique. Une introduction philosophique, Paris: Flammarion. 
Bohm D. and Hiley B. 1993. The Undivided Universe. London, New York: Routledge.

Briggs J. and Peat D. 1984. Looking Glass Universe. The Emerging Science of Wholeness. New York: Touchstone.

Clauser J. F., Horne M. A., Shimony A. and Holt R. A. 1969. "Proposed Experiment to Test Local Hidden-Variable Theories." Phys. Rev. Lett. 23: 880.

Cohen-Tannoudji C., Diu B. and Laloë B. 1997. Mécanique quantique, Paris : Hermann, collection Enseignement des Sciences.

Damasio A. 1999. The Feeling of what happens: Body and Emotions in the making of Consciousness. New York : Harcourt Brace. French trad. : Le sentiment même de soi. Corps, émotions, conscience. Odile Jacob. Paris, 1999.

Dennet D. 1991. Consciousness Explained. Boston : Little Brown. French trad., P. Engels, La Conscience expliquée. Paris, Odile Jacob, 1993.

Flanagan, O. 1994. Consciousness Reconsidered, Cambridge (USA): MIT press.

Freud, S. 1895. Studies on Hysteria. Standard Edition, vol. 2, Hogarth Press, 1955.

Groddeck G 1923. Le livre du ça. Paris : Gallimard 1973.

JanssenT. 2006. La solution intérieure. Paris : Fayard.

Jung C. G. 1988. Synchronicité et Paracelsica. Paris : Albin Michel.

Koch C. 2006. A la recherche de la conscience. Paris: Odile Jacob (French trad. from The Quest for Consciousness 2004 .

Landeau L. J. 1987. «Experimental tests of general quantum theories ». Lett. Mathematical Physics 14 : 33-40. 
Leopold D. A. and Logothetis N. K. 1996. «Avtivity changes in early visual cortex reflects monkeys' percepts during binocular rivalry ». Nature, $379: 549-553$.

Libet B. 1985. "Unconscious cerebral initiative and the role of conscious will in voluntary action." Behavioral and Brain Sciences, 8:529-566.

Mattuck R. 1980. «Une théorie quantique de l'interaction entre la conscience et la matière. » In Science et Conscience, les deux lectures de l'univers. Paris : Stock.

Mc Taggart J. 1908. "The Unreality of Time”, Mind , 17: 457-474.

Meier C.A. 1988."Science and synchronicity.” Psychological Perspectives 19 : 320-324.

Minsky M. 1988. La Société de l'Esprit, Paris : InterEditions.

Noë A. and Thompson E. 2004. «Are there Neural Correlates of Consciousness ?», Journal of Consciousness Studies, 11 : 3-28.

Pauli W. 1994. Writings on Physics and Philosophy, ed. by C.P. Enz and K. von Meyenn. Berlin: Springer.

Piaget J. 1975. L'équilibration des structures cognitives : problème central du développement. Paris : PUF.

Primas H. 2003. « Time-entanglement between mind and matter ». Mind and Matter, $1: 81$ 119.

Primas H. 2010. "Complementarity of Mind and Matter", in Recasting Reality, Atmanspacher $\mathrm{H}$ and Primas H. editors.

Ribot T. 1899. Les maladies de la personnalité. Paris: Alcan.

Römer H 2006. “Complementarity of process and substance.” Mind and Matter, 41 :69-89. 
Russell B. 1921. The Analysis of Mind. The Macmillian Company and George Allen \& Unwin Ltd, and .New York: Cosimo, 2004.

Schrödinger, E. 1935. "Discussion of Probability Relations Between Separated Systems", Proceedings of the Cambridge Philosophical Society, 31: 555-563; 32 : 446-451 (1936).

Searle J.R. 1997. The Mystery of Consciousness. London : Granta Books publisher.

Sperry R. 1983. Science and Moral Priority, New York: Columbia University Press.

Spinoza B. 1677. Ethique. Oeuvres de Spinoza, traduction Emile Edmond Saisset. Charpentier 1842.

Tononi G. 2007. "The Information Integration Theory of Consciousness" in The Blackwell Companion To Consciousness. Edited by Max Velmans and Susan Schneider. Oxford : Blackwell Publishing.

Uzan P. 2011. Conscience et Physique Quantique. Paris :Vrin, collection Mathesis, in print.

Walker E. H. 1975. Foundations of paraphysical and parapsychological phenomena. Quantum physics and parapsychology, New York: Laura Oteri Ed.

Yovel Y. 1989. Spinoza and Other Heretics: The Marrano of Reason, Volume 1. Princeton: Princeton University Press.

Zurek W.H. 1981. « Pointer basis of quantum apparatus: Into what mixture does the wave packet collapse? »Phys. Rev. D24 : 1516.

- 1982. «Environment-induced superselection rules ». Phys. Rev. D26 :1862. 